\title{
Long-Term Changes of Central Ocular Motor Signs in Patients with Vestibular Migraine
}

\author{
Hermann Neugebauer ${ }^{a}$ Christine Adrion ${ }^{b}$ Miriam Glaser ${ }^{a}$ Michael Strupp ${ }^{a}$ \\ ${ }^{a}$ Department of Neurology and the German Dizziness Center (IFB ${ }^{\mathrm{LMU}}$ ), and ${ }^{\mathrm{b}}$ Department of Medical Informatics, \\ Biometry and Epidemiology, University of Munich, Munich, Germany
}

\section{Key Words}

Vestibular migraine - Central ocular motor dysfunctions . Symptom-free interval $\cdot$ Vertigo $\cdot$ Vestibulo-ocular reflex

\begin{abstract}
Background: A high percentage of patients with vestibular migraine (VM) were reported to have central ocular motor dysfunctions (COMD) in the symptom-free interval. Since VM is a chronic disorder, it is of interest if COMD worsen over time. Methods: Thirty VM patients and 11 control patients participated in an 8-year-long observational study with follow-up. All patients underwent standardized neuro-ophthalmological and neuro-otological examinations at the initial presentation and at follow-up. The primary outcome measure was change in COMD at follow-up. Results: In general, COMD in the symptom-free interval were subtle, consisting of a saccadic horizontal and/or vertical pursuit. At follow-up, significantly more patients with VM had COMD $(p=$ 0.015). The prevalence of COMD increased from 20 to $63 \%$ in VM patients. Further, the presence of COMD at follow-up had a positive predictive value of $90.5 \%$ for the diagnosis of VM. Successful treatment with prophylactic migraine medication seems to prevent the development and worsening of COMD ( $p=0.008)$. Conclusion: Mild COMD occur in a large
\end{abstract}

percentage of VM patients during the course of the disease. The presence of COMD is predictive of VM in patients with recurrent attacks of vertigo. Prophylactic migraine medication seems to attenuate the development and progression of COMD.

Copyright $\odot 2012$ S. Karger AG, Basel

\section{Introduction}

During the last decade, vestibular migraine (VM) has been acknowledged to be a relevant clinical entity not only in dizziness units. With a lifetime prevalence of $1 \%$, VM is the most common cause of episodic vertigo [1]. Recurrent attacks of moderate to severe vertigo are strongly associated with migraine-like headache and/or migrainous symptoms such as phonophobia, photophobia, or aura. In 2001, Neuhauser et al. [2] proposed criteria for establishing a diagnosis of VM on the basis of the patients' presenting symptoms and medical history. However, because clinical presentation is unspecific, an accurate diagnosis is often made only late in the course of the disease [3,4]. A clinical test that reliably predicts VM in an early stage would be helpful.

\section{KARGER}

Fax +4161306 1234

E-Mail karger@karger.ch

www.karger.com
(C) 2012 S. Karger AG, Basel

0014-3022/13/0692-0102\$38.00/0

Accessible online at:

www.karger.com/ene
Hermann Neugebauer, MD

Center for Stroke Research Berlin (CSB)

Charité - Universitätsmedizin Berlin, Campus Virchow Klinikum

Augustenburger Platz 1, DE-13353 Berlin (Germany)

E-Mail Hermann.Neugebauer@charite.de 
Table 1. Distribution of age and sex between the study groups (mean $\pm \mathrm{SD}$ )

\begin{tabular}{lllll}
\hline & $\mathrm{dVM}$ & $\mathrm{pVM}$ & $\mathrm{dVM}+\mathrm{pVM}$ & No VM \\
\hline Total & 18 & 12 & 30 & 11 \\
Age, years & $53 \pm 12.7$ & $61.2 \pm 8.7$ & $56.2 \pm 11.9$ & $63.1 \pm 10.0$ \\
Mean follow-up, years & $7.9 \pm 1.4$ & $8.1 \pm 1.7$ & $8.0 \pm 1.5$ & $8.0 \pm 1.5$ \\
Females/males & $14 / 4$ & $4 / 8$ & $18 / 12$ & $8 / 3$ \\
\hline
\end{tabular}

$\mathrm{dVM}=$ Definite VM; pVM = probable VM; $\mathrm{dVM}+\mathrm{pVM}=\mathrm{VM} ;$ No VM = control group.

A large percentage of patients with VM have been reported to have central ocular motor dysfunctions (COMD; 18.8-65\%) such as saccadic pursuit, spontaneous or gazeevoked nystagmus, and positional nystagmus [5-7]. The high incidence (65\%) found by Dieterich and Brandt [5] is in line with prior studies that examined migrainous patients with vertigo. Kayan and Hood [6] described persisting ocular motor derangements in $77.5 \%$ of their patients, which were either central $(18.8 \%)$, peripheral $(28.8 \%)$, or inconclusive $(30 \%)$ in origin. Bir et al. [7] found electronystagmography (ENG) abnormalities in $58 \%$ of patients with migraine and vertigo. In contrast, current studies that included patients with migraine and vertigo according to the Neuhauser criteria showed different results. One study reported central ocular motor disturbances in $16.6 \%$, while two other studies reported central ocular motor signs in only about $8 \%$ of patients with VM [8-10].

Since VM is a chronic disorder with recurrent attacks of vertigo, the question arises as to whether the reported COMD worsen over time. From a diagnostic and pathophysiologic point of view it would be interesting to determine both the presence and the development of COMD over the course of the disease. We therefore performed a long-term study on 30 patients with VM who underwent a standardized examination of ocular motor functions at the time of diagnosis and at follow-up. Control patients were those who presented to our dizziness unit in the same time frame and had had an initial diagnosis of VM, but a re-diagnosis during follow-up of vestibular disorders other than VM.

\section{Patients and Methods}

\section{Patients}

Patients diagnosed to have VM in the period from 1997 to 2001 were identified in the database of our dizziness unit. Thirty patients presented for a follow-up examination and were included in the study. The original diagnoses of VM were revised according to the criteria of Neuhauser et al. [2] to 'definite VM' and 'probable VM'. Eleven age- and sex-matched patients were included in the control group. These patients had presented to our dizziness unit in the same period with similar symptoms and an initial diagnosis of VM but at follow-up the diagnosis had been revised (table 1). Except for ocular motor dysfunctions, all patients had a normal neurological status. Routinely done brain imaging in all patients did not reveal any causative lesions. The control group was chosen to determine the appropriate diagnosis for episodic vertigo. We did not choose patients with central vestibular disorders other than VM, because they usually present with different ocular motor disturbances and are most often diagnosed by the presence of additional neurological signs and abnormal brain imaging.

The diagnosis of migraine was made according to the IHCD-2 (International Headache Society Classification Subcommittee; 2004) [11] during a face-to-face interview and on the basis of a short questionnaire. Vertigo was not considered an aura symptom for the diagnosis of migraine with aura.

A standardized history was taken from all patients including characteristics of vertigo like frequency, duration, intensity and postural imbalance during the attack, associated migrainous symptoms (headache, photophobia, phonophobia, visual and other auras), a past medical history of migraine according to the International Headache Society and a family history of migraine headache. The treatment response to prophylactic migraine medication was also evaluated. A positive response was defined as a more than 50\% reduction of vertigo frequency or intensity from baseline. Patients were asked to estimate the effect of reduction in percent on an analog scale.

\section{Methods}

All patients underwent identical, standardized, complete neurological, neuro-ophthalmological and neuro-otological examinations when presenting and at follow-up. They included examination for spontaneous nystagmus with Frenzel's goggles, gaze-evoked nystagmus, smooth pursuit, saccades, optokinetic nystagmus, visual fixation suppression of the vestibulo-ocular reflex (VOR), rebound nystagmus, head-shaking nystagmus, and the Halmagyi-Curthoys head-impulse test. The subjective visual vertical as well as eye position in the roll plane were also determined with a scanning laser ophthalmoscope. The results of the follow-up were compared with records from the initial presentation. 
Table 2. Central ocular motor signs in the symptom-free interval in patients with dVM and pVM versus no VM patients at initial presentation and follow-up

\begin{tabular}{|c|c|c|c|c|c|c|}
\hline & \multicolumn{3}{|c|}{ Initial presentation } & \multicolumn{3}{|c|}{ Follow-up } \\
\hline & $\mathrm{dVM}$ & $\mathrm{pVM}$ & no VM & $\mathrm{dVM}$ & $\mathrm{pVM}$ & no VM \\
\hline Normal & 13 & 11 & 10 & 4 & 7 & 8 \\
\hline Strabismus & 0 & 2 & 0 & 0 & 2 & 0 \\
\hline Central ocular motor disturbance ${ }^{a}$ & 5 & 1 & 1 & 14 & 5 & 2 \\
\hline Saccadic vertical pursuit & 5 & 1 & 0 & 12 & 5 & 1 \\
\hline Saccadic horizontal pursuit & 3 & 1 & 1 & 8 & 4 & 2 \\
\hline Cancellation of VOR & 0 & 0 & 0 & 1 & 0 & 1 \\
\hline Unilateral gaze-evoked nystagmus & 0 & 0 & 4 & 1 & 2 & 1 \\
\hline Dissociated gaze-evoked nystagmus & 1 & 1 & 1 & 0 & 0 & 2 \\
\hline Spontaneous nystagmus $\left(>5^{\circ} / \mathrm{s}\right)^{\mathrm{b}}$ & 1 & 0 & 0 & 0 & 1 & 0 \\
\hline Deviation of the subjective visual vertical $\left(> \pm 2.5^{\circ}\right)^{\mathrm{c}}$ & 0 & 0 & 1 & 1 & 0 & 2 \\
\hline
\end{tabular}

$\mathrm{dVM}=$ Definite VM; pVM = probable VM; no VM = control group.

${ }^{a}$ Multiple answers possible. ${ }^{\mathrm{b}}$ In combination with other central ocular motor signs and/or a directional preponderance in caloric testing. ${ }^{c}$ In combination with other central ocular motor signs.

ENG and bithermal caloric irrigation were performed in all patients who were examined by ENG at initial presentation, and the mean peak slow-phase velocity (MSPV) was determined. Vestibular paresis was defined as either an asymmetry of more than $25 \%$ between the right-sided and left-sided response or a unilateral response with a MSPV $\left(30^{\circ}+44^{\circ}\right)$ of less than $10^{\circ}$ s.

\section{Statistical Analysis}

Statistical analyses included frequency and descriptive statistics. All calculations were performed using the R software package, version 2.11.1 (www.r-project.org). Fisher's exact test was applied to compare proportions. Nonparametric procedures were used to assess differences in quantitative measurements. Results are given as mean \pm SD. For statistical analysis, patients with definite VM and those with probable VM were pooled (VM group) and compared with the control group, i.e. patients without VM.

\section{Results}

Eighteen patients fulfilled the diagnostic criteria for definite VM and 12 for probable VM. Vertigo in the control group was due to Meniere's disease in 2, benign paroxysmal positional vertigo in 3 , unilateral peripheral vestibular deficit most likely due to vestibular neuritis in 2 , phobic postural vertigo in 2, bilateral vestibulopathy in 1 and orthostatic hypotension in 1.

Patients with definite VM were $53 \pm 12.7$ years old, patients with probable VM 61.2 \pm 8.7 years old, and patients in the control group $63.1 \pm 10.0$ years old (mean $\pm \mathrm{SD})$. Despite the slightly younger age of patients with definite VM, there was no statistically significant difference in age among the three groups ( $p=0.076$, KruskalWallis test). Likewise, the small difference in sex was also not significant (0.716, Fisher's exact test). The follow-up time was $7.9 \pm 1.4$ years for patients with definite VM, $8.1 \pm 1.7$ years for patients with probable VM, and $8.0 \pm$ 1.5 years (mean $\pm \mathrm{SD}$ ) for the control group.

Findings in the neuro-ophthalmologic examination (see table 2) were classified as central ocular motor disturbances by our team of orthoptists, who had examined the patients over the years in a standardized fashion. Central ocular motor disturbances were documented if ocular motor abnormalities were clearly central in origin, e.g. a disturbance of smooth pursuit. Unspecific signs such as isolated unilateral gaze-evoked nystagmus and dissociated gaze-evoked nystagmus or isolated deviations of the subjective visual vertical without further central ocular motor signs were classified as normal.

In general, central ocular motor signs were subtle, consisting of a saccadic horizontal and/or vertical pursuit. Unspecific central ocular motor signs were infrequent and mainly seen in the control group. Central ocular motor signs were detected at initial presentation in 6 patients with VM (definite and probable) and in 1 patient of the control group ( $\mathrm{p}=0.65$, Fisher's exact test). Nineteen patients with VM presented with central ocular motor signs at follow-up compared to 2 patients of the control group ( $p=0.015$, Fisher's exact test). Thus, the central 
Table 3. Clinical characteristics of patients with VM (definite and probable migrainous vertigo) versus no VM

\begin{tabular}{|c|c|c|c|}
\hline Clinical features & No VM & VM & $\begin{array}{l}\mathrm{p} \text { value (Fisher's } \\
\text { exact test) }\end{array}$ \\
\hline \multicolumn{4}{|c|}{ COMS initial presentation } \\
\hline Yes & 1 & 6 & \multirow[t]{2}{*}{0.651} \\
\hline No & 10 & 24 & \\
\hline \multicolumn{4}{|l|}{ COMS follow-up } \\
\hline Yes & 2 & 19 & \multirow[t]{2}{*}{0.015} \\
\hline No & 9 & 11 & \\
\hline \multicolumn{4}{|l|}{ Change in COMS } \\
\hline Yes & 1 & 13 & \multirow{2}{*}{0.064} \\
\hline No & 10 & 17 & \\
\hline \multicolumn{4}{|c|}{ Migraine according to the IHS } \\
\hline Yes & 2 & 21 & \multirow[t]{2}{*}{0.005} \\
\hline No & 9 & 9 & \\
\hline \multicolumn{4}{|c|}{ Family history of migraine } \\
\hline Yes & 2 & 21 & \multirow[t]{2}{*}{0.005} \\
\hline No & 9 & 9 & \\
\hline \multicolumn{4}{|c|}{ Migrainous symptoms during vertigo } \\
\hline Yes & 5 & 26 & \multirow[t]{2}{*}{0.013} \\
\hline No & 6 & 4 & \\
\hline \multicolumn{4}{|c|}{ Intensity of vertigo } \\
\hline Low & 4 & 0 & \multirow[t]{3}{*}{0.004} \\
\hline Moderate & 5 & 15 & \\
\hline Severe & 2 & 15 & \\
\hline \multicolumn{4}{|l|}{ Gait disturbance } \\
\hline Yes & 6 & 20 & \multirow[t]{2}{*}{0.491} \\
\hline No & 5 & 10 & \\
\hline \multicolumn{4}{|c|}{ Response to prophylactic migraine medication } \\
\hline Yes & 0 & 18 & \multirow[t]{2}{*}{0.001} \\
\hline No & 7 & 7 & \\
\hline
\end{tabular}

COMS = Central ocular motor signs; IHS = International Headache Society.

ocular motor signs worsened over the years in 13 patients with VM and in 1 patient of the control group ( $p=0.064$, Fisher's exact test). Expressed in terms of test performance parameters, the development of central ocular motor signs had a positive predictive value (PPV) of 92.86\% (95\% CI 68.53-98.73) for the diagnosis of VM. The presence of central ocular motor signs at follow-up showed a PPV for the diagnosis of VM of $90.48 \%$ (95\% CI 71.09-97.35).

Twenty-one patients with VM reported having a positive past medical history of migraine headache (11 with and 10 without aura) in contrast to only 2 patients in the control group ( 1 with and 1 without aura; $p=0.005$, Fisher's exact test). Twenty-six patients with VM experienced migrainous symptoms during attacks of vertigo com-

Long-Term Changes of Central Ocular Motor Signs in Patients with VM pared to only 5 patients in the control group $(\mathrm{p}=0.013$, Fisher's exact test). Patients suffering from VM showed significantly more migrainous symptoms than patients of the control group ( $p=0.017$, Cochrane-Armitage trend test).

Four patients in the control group perceived the intensity of vertigo as low, whereas none of the VM patients experienced only a low intensity of vertigo. Five patients of the control group reported a moderate intensity compared to 15 patients with VM. Only 2 control patients reported severe vertigo in contrast to 15 patients in the VM group ( $\mathrm{p}=0.004$, Fisher's exact test).

Eighteen patients with VM reported a positive response to prophylactic migraine medication, whereas 7 did not improve and 4 did not take the prescribed medication. Fifteen patients took beta-blockers as prophylactics, 2 sodium valproate, and 1 patient flunarizine. Five of the 11 patients in the control group were also treated with prophylactic migraine medication early in the course of their disease: 4 received beta-blockers and 1 sodium valproate. As expected, none of them improved over time. A comparison of both groups showed a significant difference in the response to prophylactic migraine medication in favor of the patients with VM ( $\mathrm{p}=$ 0.001 , Fisher's exact test). Clinical characteristics and statistical analysis are summarized in table 3.

In view of the influence of prophylactic migraine medication on the development of central ocular motor disturbances in patients with VM, patients with a positive response to migraine medication were compared with patients who did not benefit from prophylactic treatment. Fourteen of 18 patients $(77.8 \%)$ who responded to treatment showed stable ocular motor characteristics compared to 3 of 12 patients (25\%) who did not respond. The remaining 4 patients (22.2\%) and 9 patients (75\%), respectively, developed additional ocular motor disturbances. This difference was statistically significant ( $\mathrm{p}=$ 0.008 , Fisher's exact test).

ENG testing was performed in 24 patients at initial presentation and in 18 patients at follow-up in the VM group. None of the patients had a canal paresis at initial presentation. At follow-up, 6 patients showed an asymmetry of more than $25 \%$ between the right-sided and the left-sided response. However, 4 patients showed a normal response to either warm-water or cold-water irrigation. Thus, a definite canal paresis was found in only 2 patients. Seven patients in the control group underwent ENG testing at initial presentation and 5 at follow-up. A definite canal paresis was not documented at initial presentation, but was found in 2 patients at follow-up. A 
comparison of both groups showed no statistical difference either at initial presentation ( $p=0.492$, Fisher's exact test) or at follow-up ( $\mathrm{p}=0.156$, Fisher's exact test). Patients who had a complete ENG workup including smooth pursuit testing at initial presentation and at follow-up showed identical central ocular motor disturbances as they did in the neuro-ophthalmologic examination.

\section{Discussion}

This study showed that the prevalence of central ocular motor signs in the symptom-free interval of patients with VM increases during the course of the disease. However, these disturbances were only mild, and if initially present, did not significantly worsen over the years. In general, central ocular motor signs were subtle, consisting of a saccadic horizontal and/or vertical pursuit. Unspecific signs, such as spontaneous or unilateral gazeevoked nystagmus, were infrequent and mainly seen in the control group.

The different prevalences of central ocular motor findings are in line with previous reports. The high prevalence at follow-up agrees with previous findings [5], whereas the low prevalence at initial presentation is comparable to the results of recent studies that reported around 8 and $16.6 \%$ [8-10]. Thus, the clear-cut increase in central ocular motor findings at follow-up explains well the different results reported in earlier studies as being due to a time bias. A population bias, on the other hand, is unlikely, because the inclusion criteria were identical [2]. Furthermore, the comparable distribution of findings in our study and in other studies is indirect proof that the populations of patients were similar (e.g. percentage of peripheral vestibular deficit in our study 11 vs. $8.3-28.8 \%$ in previous studies) $[5,6,8,9,12]$.

Despite the diagnostic criteria published by Neuhauser et al. [2], which have been proven valid and reliable [13], the diagnosis of VM can still be difficult. It is current practice to exclude relevant differential diagnoses in patients with suspected VM, to observe their response to migraine medication, and to follow up the patients. Mild central ocular motor disturbances are thought to point to a diagnosis of VM [5]. The data of this study show that central ocular motor signs at initial presentation do not occur more frequently in patients with VM than in patients with vertigo due to a disease other than VM, but they do occur at follow-up in significantly more patients with VM. Therefore, central ocular motor signs are of small diagnostic value initially, but their presence at follow-up reliably indicates VM. Similarly, the development of central ocular motor signs over the years is predictive of VM in the majority of cases. It is tempting to speculate that central ocular motor signs could also be of equal diagnostic value in patients who present for the first time after having suffered from recurrent attacks of vertigo for years.

Despite their increased prevalence, central ocular motor signs did not worsen during the follow-up interval in patients who initially presented with central ocular motor signs. The data do not clearly show if this observation reflects the natural course of the disease. We suspect that the stable characteristics of central ocular motor disturbances are due to the treatment effects of prophylactic migraine medication. Possible causes of VM are vasospasms within the vestibular and/or auditory labyrinth [14-16] and dysfunction of voltage-sensitive ion channels or neurotransmitters alone or in combination with spreading depression [5, 17-22]. Just as the pathophysiological mechanisms underlying VM have not yet been unraveled, it is also unclear how migraine prophylactics influence the course of the disease. However, patients who were successfully treated with prophylactics in our study experienced a reduction in attack frequency, duration and intensity, and were more likely to show stable ocular motor characteristics, whereas patients who failed to respond to treatment, for whatever reasons, were more likely to develop ocular motor signs at follow-up. It is important to note that the small size of the study and the retrospective acquisition of treatment parameters such as dose, duration and adherence limit our ability to infer treatment effects.

We have to emphasize that uncritical standard interpretation of mild ocular motor signs - essentially mild smooth pursuit disorder - might be problematic. Because smooth pursuit much depends on attention, good cooperation of the patient is a critical precondition. Otherwise smooth pursuit is even saccadic in normal subjects. Some authors suggest testing for cancellation of the VOR during combined eye-head tracking to infer on smooth pursuit if a direct examination is difficult to achieve. As a general rule, lesions disrupting smooth pursuit impair VOR cancellation in the same way [23]. In contrast, degenerative diseases of the brainstem and cerebellum show a discrepancy between the poor performance of smooth pursuit and a superior performance of VOR cancellation [24]. Therefore, the well-preserved function of VOR cancellation and the poor smooth pursuit observed in our cohort do not suggest poor cooperation, but do point to- 
wards central infratentorial dysfunction of the ocular motor system.

In conclusion, our findings indicate that although central ocular motor signs are only mild and stable, they may be of diagnostic value for VM for the critical clinician. Patients with recurrent attacks of vertigo readily exhibit an interruption of smooth pursuit but preserved VOR cancelation during the ocular motor examination; this makes a diagnosis of VM most likely. In contrast, ocular motor findings other than interrupted saccadic pursuit at both the initial presentation and at follow-up point to a different diagnosis.

\section{References}

$>1$ Lempert T, Neuhauser H: Epidemiology of vertigo, migraine and vestibular migraine. J Neurol 2009;256:333-338.

$>2$ Neuhauser H, Leopold M, von Brevern M, Arnold G, Lempert T: The interrelations of migraine, vertigo, and migrainous vertigo. Neurology 2001;56:436-441.

$>3$ Neuhauser H, Lempert T: Vertigo and dizziness related to migraine: a diagnostic challenge. Cephalalgia 2004;24:83-91.

$\checkmark 4$ Brandt T, Strupp M: Migraine and vertigo: classification, clinical features, and special treatment considerations. Headache Currents 2006;3:12-19.

5 Dieterich M, Brandt T: Episodic vertigo related to migraine (90 cases): vestibular migraine? J Neurol 1999;246:883-892.

$\checkmark 6$ Kayan A, Hood JD: Neuro-otological manifestations of migraine. Brain 1984;107:11231142.

7 Bir LS, Ardiç FN, Kara CO, Akalin O, Pinar HS, Celiker A: Migraine patients with or without vertigo: comparison of clinical and electronystagmographic findings. J Otolaryngol 2003;32:234-238.

$>8$ Teggi R, Colombo B, Bernasconi L, Bellini C, Comi G, Bussi M: Migraino us vertigo: results of caloric testing and stabilometric findings. Headache 2009;49:435-444.

$>9$ Celebisoy N, Gökçay F, Sirin H, Biçak N: Migrainous vertigo: clinical, oculographic and posturographic findings. Cephalalgia 2007; 28:72-77.
10 Vitkovic J, Paine M, Rance G: Neuro-otological findings in patients with migraine- and nonmigraine-related dizziness. Audiol Neurootol 2008; 13:113-122.

11 Headache Classification Subcommittee of the International Headache Society: The International Classification of Headache Disorders, ed 2. Cephalalgia 2004;24:9-160.

12 Cutrer FM, Baloh RW: Migraine-associated dizziness. Headache 1992;32:300-304.

13 Radtke A, Neuhauser H, von Brevern M, Hottenrott T, Lempert T: Vestibular migraine - validity of clinical diagnostic criteria. Cephalalgia 2011;31:906-913.

14 Viirre ES, Baloh RW: Migraine as a cause of sudden hearing loss. Headache 1996;36:2428.

15 Cass SP, Furman JM, Ankerstjerne K, Balaban C, Yetiser S, Aydogan B: Migraine-related vestibulopathy. Ann Otol Rhinol Laryngol 1997;106:182-189.

16 Lee H, Lopez I, Ishiyama A, Baloh RW: Can migraine damage the inner ear? Arch Neurol 2000;57:1631-1634.

17 Kim JS, Yue Q, Jen JC, Nelson SF, Baloh RW: Familial migraine with vertigo: no mutations found in CACNA1A. Am J Med Genet 1998;79:148-151.

18 von Brevern M, Ta N, Shankar A, Wiste A, Siegel A, Radtke A, Sander T, Escayg A: Migrainous vertigo: mutation analysis of the candidate genes CACNA1A, ATP1A2, SCN1A, and CACNB4. Headache 2006;46: 1136-1141.
19 Furman JM, Marcus DA, Balaban CD: Migrainous vertigo: development of a pathogenetic model and structured diagnostic interview. Curr Opin Neurol 2003;16:5-13

20 Koo JW, Balaban CD: Serotonin-induced plasma extravasation in the murine inner ear: possible mechanism of migraine-associated inner ear dysfunction. Cephalalgia 2006;26:1310-1319.

21 Vass Z, Dai CF, Steyger PS, Jancso G, Trune DR, Nuttall AL: Co-localization of the vanilloid capsaicin receptor and substance $\mathrm{P}$ in sensory nerve fibers innervating cochlear and vertebro-basilar arteries. Neuroscience 2004;124:919-927.

22 Vass Z, Steyger PS, Hordichok AJ, Trune DR, Jancso G, Nuttall AL: Capsaicin stimulation of the cochlea and electric stimulation of the trigeminal ganglion mediate vascular permeability in cochlear and vertebro-basilar arteries: a potential cause of inner ear dysfunction in headache. Neuroscience 2001; 103:189-201.

23 Leigh RJ, Zee DS: The Neurology of Eye Movements, ed 4. Oxford, Oxford University Press, 2006, pp 334-335

24 Grant MP, Leigh RJ, Seidman SH, Riley DE, Hanna JP: Comparison of predictable smooth ocular and combined eye-head tracking behaviour in patients with lesions affecting the brainstem and cerebellum. Brain 1992;115:1323-1342. 